\title{
SYDACK - System of Diagnosis and Control in Kinesitherapy
}

\author{
Vesna Filipović \\ Study of Physiotherapy, Health Polytechnics, Mlinarska cesta 38, 10000 Zagreb \\ vesna.filipovic@zvu.hr \\ Natasa Viskić-Štalec \\ Faculty of Kinesiology University of Zagreb, Horvaćanski zavoj 15, 10000 Zagreb \\ viskic@kif.hr \\ Janez Śtalec \\ Department of Mathematics, University of Zagreb, Bijenička 30, 10000 Zagreb \\ stalec@math.hr \\ Elvis Popović \\ elvis.popovic@zg.hinet.hr \\ Saša Vuk \\ Postgraduate student - Faculty of Kinesiology University of Zagreb, Horvaćanski zavoj 15, \\ 10000 Zagreb \\ sasavukhr@vip.hr
}

\begin{abstract}
The SYDACK system of diagnosis and control in kinesitherapy has been developed. It consists of Avatar exercises - a virtual instructor, two sensors for monitoring a subject's shoulder and hip movement and computer software which combines the information from sensors placed on the subject's body with the computer. There are two ways to access the data: (1) graphically, as feedback information about the deviation of movement between the subject and the Avatar dispatched to the subject in real time and (2) via numerical data about subject's postural status and kinesitherapy process, expressed in angles, stored in a database for future processing and analysis.
\end{abstract}

Keywords. virtual reality, movement monitorring sensor, kinesitherapy

\section{Introduction}

Kinesitherapy, like part of physiotherapy, to achieve natural function and natural human motion, is based on prompt and detailed diagnosis. More recently, information technology is being more and more utilised to enhance human posture estimation and monitoring of kinesitherapy effects. Some interpretations can be followed in research papers. For example, Girone et al. [1] and Girone et al. [2] apply a combined platform system of virtual reality (VR) and base force analysis by measuring angles through $\mathrm{x}, \mathrm{y}$ and z-axis. The patient follows a VR generated exercise program displayed on a computer screen and the force exerted by the patient is then measured. The exercises are adapted to the patient's diagnosis and therapy. Angles of ankle movements are stored in a database and the decision to undertake further therapy is based on the collected results.

Hollerbach et al. [3] monitor and analyze a subject's body movements using the SARCOS system which in real time records the mechanical force of walking on a movable platform. The subject imitates the image displayed on a wall projected by three slide projectors.

Christensen et al. [4] use the SARCOS system to analyse inertial force during movement acceleration and compare base forces with the standard forces in the measure system. Vijayakar et al. [5] use the SARCOS system to analyse head and torso rotation forces during walking.

Checcacci et al. [6] upgrade the same system by connecting the body with measuring instruments. They emphasize the importance of the relationship between the forces of the hip and pelvic joints with the spine.

A group of authors at the University of Kinesiology in Zagreb combine virtual reality, technology that monitors movement (i.e. sensors) and actual human posture which then enables capture of accurate data and storage in real time. It is also important that the subject receives feedback information regarding their required movement and the deviation from the desired movement via a computer screen.

The published research analysed the force of movement (Hollerbach, 2000. Vijayakar, 2002, 
Checcaci, 2003) but the of SYDACK is to follow the quality of movement during therapy (in practice, postural adaptation and movement with minimal power).

Avatar and sensor system together are a simple form of visualization where the picture explains exercise as a complex shape and rhythm. Examinee observes his mistakes during the execution of his movement and accomplishing his posture.

The basic problem of this research is to discover whether such a system can be applied for diagnostic and other evaluation purposes within kinesitherapy and kinesiology. The research was conducted with the aim to calculate the relevance and the applicability of this new system for therapy process evaluation.

\section{The Basic SYDACK system of diagnosis and control in kinesitherapy construc- tion and application}

The SYDACK system was constructed at the University of Kinesiology in Zagreb to register and control a subject's movements and then estimate their postural status (as a part of the scientific research Virtual Reality in Kinesiology; project leader was N. Viskić-Štalec, Phd of Science, done for the Croatian Ministry of Science, Education and Sports No: 0034216). A sensor system for monitoring the subject's movement, a 25 virtual instructor's therapy exercises and finally the computer software program which combines both the information from the sensors with the virtual instructor's movements were all created. The paper presents a results analysis example of one subject ${ }^{1}$ in one exercise.

The next phase of this research is application of the SYDACK in physiotherapy to pathological postural adaptation of the adolescent idiopathic scoliosis.

In addition, the use of SYDACK system in sport and other kinesiological activities is also possible.

\subsection{Measuring method in the exercise of lifting hips while sitting}

Sensors are fastened on the subject's body: the first sensor marks shoulder line by artic. acromioclavicularae left and right shoulder axis and the second sensor indicates pelvic line by lumbosacral fusion. The subject is barefoot, with her feet spread at hip width, the pelvis is supported on a chair's front edge, the subject's palms resting on her upper legs, sitting upright on the chair in front of a computer screen and imitating the Avatar's (virtual instructor's) exercises.

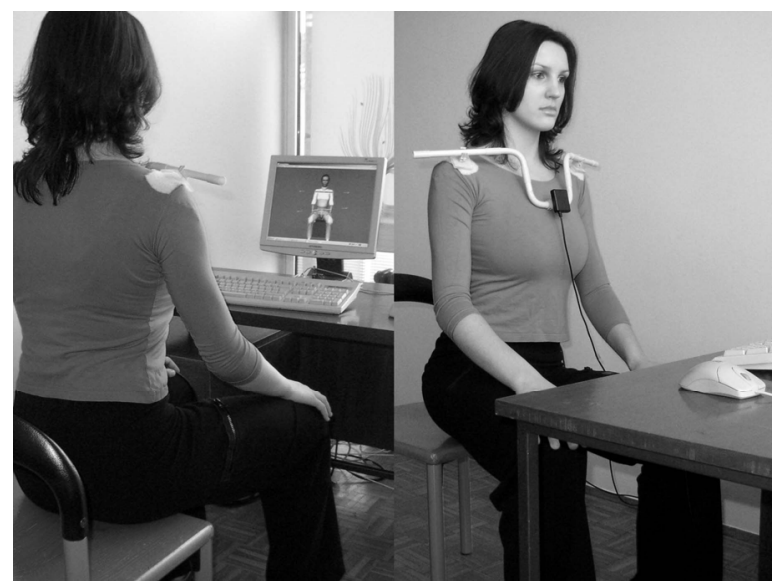

Figure 1. Subject wearing sensors is exercising in front of PC.

Exercise: The subject maintains static shoulder cuff axis movement whilst performing interchanging left/right hip elevation movement. Movements are carried out in three series of ten elevations ( 15 second pause after every series of 10 elevations).

Measuring: Measuring begins after the sensor's central box keypad is pressed (the subject repeats the exercise five times before measuring, during which time she performs the movement and adjusts her pace accordingly with the Avatar's pace). A computer database stores in real time all the subject's sensor registered movements. The counting automatically stops after the tenth routine has been performed.

Data: Sensors record the measured deviation of the shoulder and pelvis axis from the zero position $^{2}$ of the exercise:

\footnotetext{
${ }^{2}$ The zero position of the exercise is the subject's start position, no matter whether it is ideal or not. Hence monitoring of the transformations of the subject's who have abnormal postural body adaptation is made possible.

\footnotetext{
${ }^{1}$ The system was performed on 40 female students of the University of Kinesiology and 40 female students of the School of Physiotherapy in Zagreb with the aim to test instruments' measurement characteristics.
} 
- Graphically the shoulder and pelvis axis on the Avatar's body are represented by red lines. The subject can monitor the deviation of her axis (white broken lines) from the Avatar's axis (red lines) on the computer screen and therefore correct her movements.

- Displayed on the lower part of the computer screen before the measuring period are four position indicators showing the subject's shoulder to pelvis axis deviation from zero. The first two demonstrate $\mathrm{x}$-axis movement horizon-tal shoulder position deviation and $y$ axis movement - vertical position deviation (sensor 1). The other two indicators in the same way give information about pelvic position (sensor 2). This information is stored in a database. The software registers the subject's movement data every 0.045 seconds.

- Zero position deviations (expressed in angles), for ten repetitions in every kinesitherapy exercise, are stored in a database for every subject separately and can be graphically represented in the form of different statistically processed data curves or charts for later analysis. Based on the data processing and analysis about the subjects postural status and movement transformation quality, conclusions can be made about the subjects motor function learning process

\subsection{SYDACK system construction}

The purpose of this basic system is to a) collect the data from sensors attached on the subject's body, b) graphically present them on a screen and then c) process and store the information in a database.

The software records data regarding the amount of deflection by the sensor towards two axes: $\mathrm{x}$ and $\mathrm{y}$. As sensors are based on a pendulum, the azimuth (angled in position to the vertical plane in the direction of the field of gravitation) can't be measured.

Every 0.045 seconds, data from the sensor reaches the computer in analog form via a game port on the computer sound card and then digitises it. The Windows multimedia library is used to access the game port. The sensor is handled as a double joystick, each sensor having two sticks and one button. The software has its own calibration, specific for the aforementioned sensor device. Because the sensor feature is not linear, the software makes it linear according to the parameters of its calibration.
The computer software presents sensor indicators on the exercising figure for each exercise performed. The sensor provides deviation angle information whilst position is gained from the adequate tracking file for each animation.

Indicators can be set in mirror mode so that the subject perceives them as her own image reflected in the mirror.

Measuring is preceded by countdown and is activated with the sensor's central box keypad which is adjoined to the joystick button. In real time, the information is stored in a text file to achieve easier manipulation, analysis and overview utilising any word processor.

Measuring stops automatically after 10 exercise cycles have been completed and can also be stopped by pressing the sensor's central box keypad. A database stores: the name of the exercise (based on the name of the animation), the date and time of measurement, the duration of the exercise and other relevant information.

A graphical display uses the Windows media subsystems for animation decompression, DIB bitmaps for animation display and the GDI+ system for the presentation of all other information and parameters. Microsoft Windows $\mathrm{XP}$ is the recommended operating system. The necessary visualisations performed by the GDI+ graphical subsystem. Drawing is always processed in the memory bitmap.

Computer generated graphics that create and animate the Avatar instructor 3D object are performed by a special software program called Poser4 [7]. A distinctive feature of the virtual instructor simulation is the animation of targeted and preferred movements.

After the desired Avatar model has been created, the display model style called texture shaded is defined. Skin texture, certain colour and light combinations are prescribed to each body part thus achieving greater model authenticity. Infinite lights (default Poser lights) are used to illuminate all parts of the scene.

Both orthogonal and 3D (main) cameras, which serve to create the final image, are used to create and animate the model.

The background scene is grey. The SYDACK system is planned to be improved with an augmented reality creation option.

The avatar model is put into the desired pose by using the editing tools, which are manually set in the desired position, and by using numerical parameters of the desired size change 
(parameter dials). In this way the desired object pose is obtained in just one picture (frame).

Two different poses, between which parameters interpolate to achieve a smooth, continuous movement, defined by the keyframe animation, need to be created in the animation or the moving of the whole or a part of the body effect.

The animation is coded and compressed by the codec. The preferred resolution is $640 \times 480$ pixels, adjusted to the full screen mode.

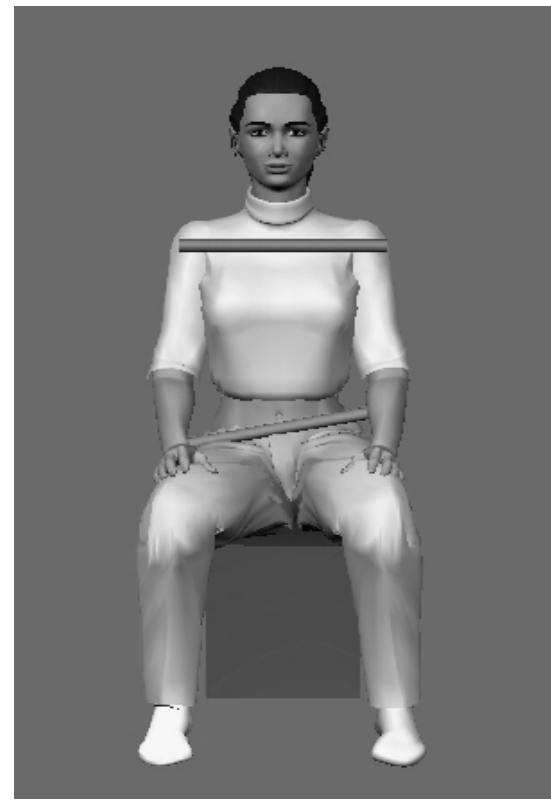

Figure 2. Avatar - the exercise of lifting hips

\section{The presentation of subject's results in the exercise of lifting hips while sitting}

The motor function exercise hip lifting requires a selected pelvic movement while maintaining a static shoulder cuff, this actually is a change of the usual pelvis and shoulder cuff movement pattern. This exercise was therefore unfamiliar to the majority of subjects.

With the help of the virtual instructor and the data displayed on the computer screen, subject managed to perceive the key parameters necessary for the effective pelvic and shoulder cuff motor behaviour pattern change and successfully completed the task.

Sensor 1 measured the subject's shoulder cuff activity; the shoulder symmetry was recorded (the deviation ranging 2-5 degrees represents a negligible dynamics value within the given static conditions). Figure 3. demonstrates the typical process of solving a new motor function task; after the third attempt, the subject succeeded to coordinate the movements of one shoulder with the opposite hip.

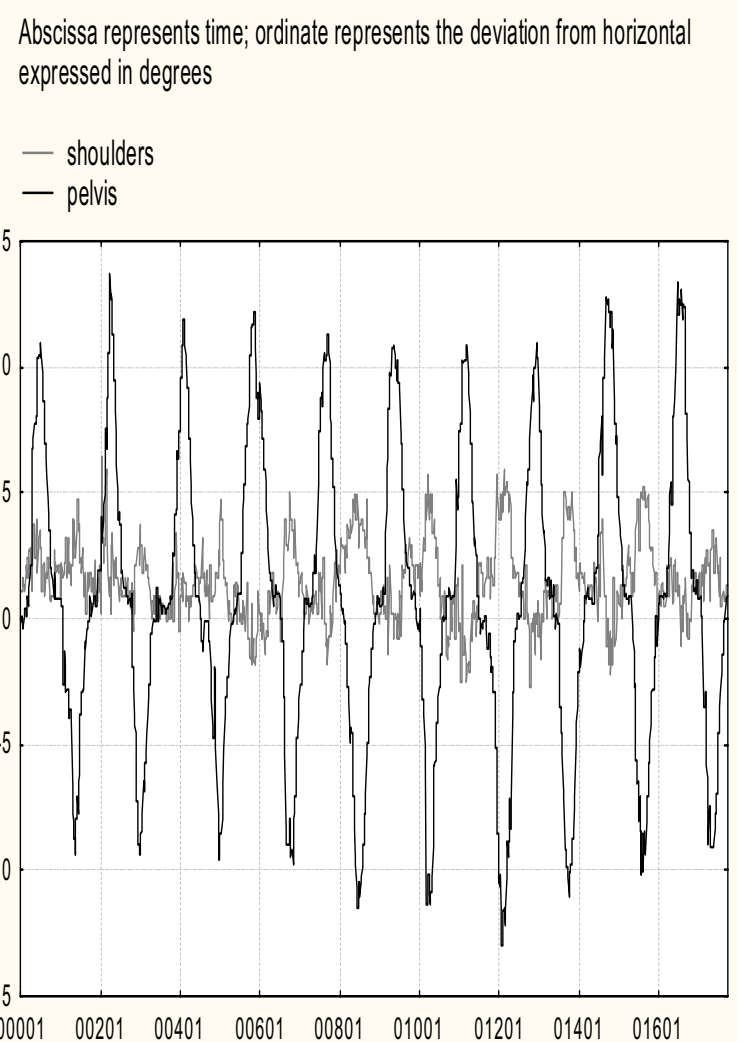

Figure 3. Intermediate left and right hip lifting (dark line) with synchronous shoulder inaction (light line) amplitude.

The results of the analysis of measuring of deviation angles from zero position recorded with sensor 2 (Figure 3.) prove that the subject's left and right hip were symmetric during pelvic elevation. The movement amplitude intermission and oscillation (levelled in the activity transfer from left to right hip), as well as a slight oscillation at the high point of the curve at the end of the elevation movement, are usual for the natural movement.

On the y-axis (Figure 4.) both body segments are in a slight deviation from the zero position. Regardless, their activity is symmetrical; the shoulder deviation range is a maximum of 5 degrees and the pelvis shifts forward (a partial inward hip rotation). Figure 4. demonstrates the $y$-axis relative symmetry during solving a new movement, realized only after the fourth repetition.

Based on subject's result data, the application of the basic SYDACK system, which combines sensors with the virtually displayed kinesitherapy task, is evident. The subject's movement quality 
can be analysed through the $\mathrm{x}$ and $\mathrm{y}$-axis amplitudes. The basic postural adaptation analysis parameter is the very body segment symmetry analysis in both: movement amplitude and in the movement repetition.
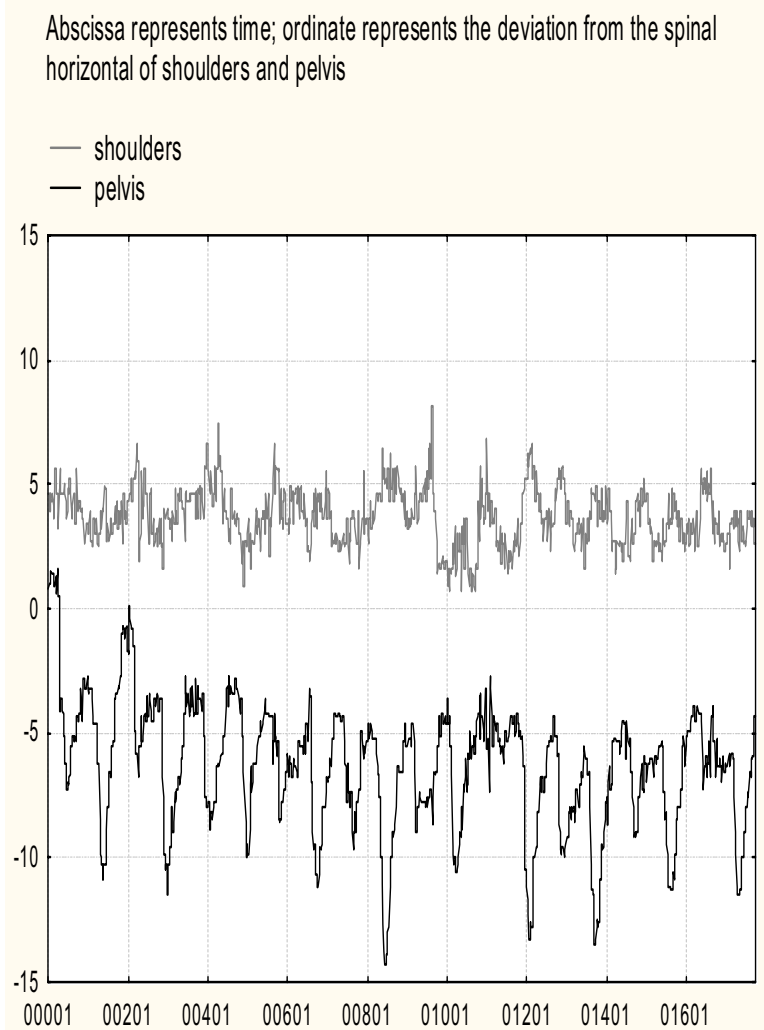

Figure 4. Intermediate left and right hip lifting (dark line) with synchronous shoulder inaction (light line) amplitude.

\section{Conclusion}

The SYDACK system was developed at the University of Kinesiology in Zagreb for the needs of kinesitherapy process evaluation, as a part of a thorough system for the movement control in sport, recreation and other kinesiology activities.

The SYDACK system consists of an animated Avatar instructor performing kinesitherapy exercises, created by the specialized software program Poser4; a set of two subject's motion detector sensors and a computer software for combining the information from the sensors placed on the subject's body with the computer.

The SYDACK system is specific because of the useful data offered to both the subject and the researcher, in real time exercise performance (visualisation). Additionally, due of the large amount obtained from the movement tracking and evaluation sensors the data are recorded and stored in a database so that they can be subsequently analysed for quality of movement.

Virtual reality technology was used for graphic representation of the subject's movement during the kinesitherapy exercises performance and the Avatar instructor's movement. The subject's task was to imitate the virtual instructor's movement on the computer screen. Two indicator systems serve as the subject and researcher's feedback information:

- Lines on the Avatar's shoulders and hips and broken lines (situated over the lines, on the Avatar), as sensor information about the subject's movement. The angles between the lines are feedback information about the accuracy of the subject's movement.

- During the measuring, four indicators of the subject's shoulder and pelvis axis deviation and the visual presentation of the angle deviation from the zero position, serving as initial system calibration, are displayed on the lower part of the computer screen.

The results of this research demonstrated the application of the SYDACK system in practice. The continuation of the research will prove the system's applicability in kinesiology and other scientific areas. Additionally, intention is application SYDACK in physiotherapy to pathological postural adaptation of the patients with adolescent idiopathic scoliosis. The main goal of the postural adaptation therapy is visualisation and detailed analysis of the movement. The continuity in following every degrees of movement to some body segments (pelvis and shoulder circle) are very important for therapist. Because of that the amplitudes of movement in $\mathrm{x}$ and $\mathrm{y}$-axis are precious and the SYDACK system enables them. They are objective indicator of momentary postural status and the results of therapy exercise.

In addition to visual evaluation during the therapy the therapist has access to detailed analysis of patient's results. Postural adaptation is the sum of functions of separate body parts. Therefore, the correct function of every part is a chain reaction in the function of the whole body.

\section{Bibliography}

[1] Girone, M., Burdea, G., Bouzit, M., Popescu, V. A Stewart platform-based system for ankle telerehabilitation. Autonomous Robots. 10 203-12. 2001. 
[2] Girone, M., Burdea, G., Bouzit, M., Popescu, V. Orthopedic rehabilitation using the „Rudgers Ankle“ interface. EuroHaptics Conference July, 2001. The University of Birmingham, UK. 2001.

[3] Hollerbach, JM., Xu, Y., Chritensen, RR., Jacobsen, SC. Design specifications for the secind generation sarcos treadport locomotion interface. Haptics Symposium, Proc. ASME Dynamic systems and control division, DSC, Orlando, 69(2) 1293-98. 2000.

[4] Christensen, RR., Hollerbach, JM., Xu, Y., Meek, SG. Inertial-force feedback for the treadport locomotion interface.
Teleoperators and virtual enviorenments. Massachusetts Institute of Technology. 91 14. 2000.

[5] Vijayakar, A., Hollerbach, J. Effect of turning strategy on maneuvering ability using the treadport locomotion interface. Teleoperators and virtual enviorenments. Massachusetts Institute of Technology. 11 114. 2002.

[6] Checcacci, D., Hollerbach, J., Hay-ward, R., Bergamasco, M. EuroHaptics conference, Dubling. 53-67. 2003.

[7] Franger, C., Stevens, L. Meta Creation Poser User Guide. MetaCreations Corporation. 1999. 